\title{
Spontaneous ventilation combined with double-lumen tube intubation in thoracic surgery
}

\author{
József Furák ${ }^{1}$ - Zsolt Szabó ${ }^{2}$
}

Received: 26 August 2020 / Accepted: 12 December 2020

(C) The Japanese Association for Thoracic Surgery 2021

\begin{abstract}
Objective We present the combination of spontaneous ventilation and double-lumen tube intubation in thoracic surgery. Methods At the beginning of the procedures, the patients with a body mass index of $\leq 30$ were relaxed for a short time, and a double-lumen tube was inserted. After the utility incision or thoracotomy, the vagus nerve was blocked (in right side in the upper mediastinum; in left side in the aorto-pulmonary window) with $3-5 \mathrm{ml}$ of $0.5 \%$ bupivacaine. The patients had a bispectral index of 40-60. After the short relaxation period, the patients were ventilating spontaneously without any cough during the manipulation.

Results Between March 10 and September 18. 2020, 26 spontaneous ventilation combined with intubation surgeries were performed: 19 uniportal video-assisted thoracic surgery (15 lobectomies, 1 segmentectomy, and 3 wedge resections) and 7 open (5 lobectomies and 1 sleeve segmentectomy, 1 wedge resection). The mean mechanical and spontaneous one-lung ventilation time was $25.5(15-115)$ and 73.3 (45-100) minutes, respectively. In 2 cases conversion to relaxation were necessary $(2 / 26 ; 7.7 \%)$. The mean maximal carbon dioxide pressure was 52.3 (38-66) Hgmm and the mean lowest oxygen saturation was 93.8 (86-99) \%. Breathing frequency ranged between 10-25/minute. The mean surgical times was 83.3 (55-130) minutes. Conclusions Spontaneous ventilation combined with intubation in video-assisted thoracic surgery or open resections is a safe method in selected patients. It can reduce the mechanical one-lung ventilation period with $76.6 \%$ and give safe airway for spontaneous ventilation thoracic procedures.
\end{abstract}

Keywords Spontaneous ventilation $\cdot$ Intubation $\cdot$ Video-assisted thoracic surgery $\cdot$ Non-intubated

\section{Introduction}

Video-assisted thoracic surgery (VATS) combined with spontaneous ventilation (SV) provides the least potential stress and fewer complications [1,2]. In the case of relaxation and mechanical ventilation, the immunological advantages of spontaneous ventilation surgery (the lower negative impact on lymphocytes and natural killer cells) are lost [3], and the disadvantages of the mechanical one-lung ventilation (OLV), such as alveolar damage (injury of the

József Furák and Zsolt Szabó contributed equally to this work.

József Furák

jfurak@gmail.com

1 Department of Surgery, University of Szeged, Semmelweis u. 8. 6725 , Szeged, Hungary

2 Department of Anesthesiology, University of Szeged, Szeged, Hungary endothelial glycocalyx and capillary shear stress, oxidative stress, and edema formation) may develop $[4,5]$. The longer the mechanical OLV, the more severe the oxidative stress [6].

From an anesthesiologist's perspective, several trials combined spontaneous ventilation with the intubation to prevent the negative side effects of relaxation and mechanical ventilation; however, none of them were fully successful [7]. To tolerate an endotracheal tube as a foreign body in the trachea without cough, the cough reflex must be stopped by blocking the vagus nerve [8]. Although the vagal block is well known in SV surgery [9], the combination of the vagus blockade and intubation is not published in the literature.

We developed a new technique, spontaneous ventilation combined with double-lumen tube intubation (SVI), to reduce the length of the mechanical OLV and provide a 'safe airway' for the SV thoracic surgeries. To the best of our knowledge, this is the first study to report this kind of surgical operation. 


\section{Methods}

\section{Patients}

Between March 10 and September 18. 2020, 26 SVI surgeries were performed (14 male; 12 female) with a mean age of 65.2 (43-80) years (Table 1.) The follow-up period lasted until September 29, 2020.

The pre-operative pulmonological examination was the same as in a VATS, non-intubated thoracic surgery (NITS), or normal open cases, and patients were not selected based on their comorbidities (Table 1). The SVI procedure and its risks were explained to each patient. Additional informed consent was obtained from all patients for which identifying

Table 1 Surgical parameters of the SVI patients

\begin{tabular}{|c|c|c|c|}
\hline & $\begin{array}{l}\text { All cases } \\
n=26\end{array}$ & $\begin{array}{l}\text { VATS SVI } \\
n=19\end{array}$ & $\begin{array}{l}\text { Open SVI } \\
n=7\end{array}$ \\
\hline Male/female & $14 / 12$ & $9 / 10$ & $5 / 2$ \\
\hline Age & $65.2(43-80)$ & $63.7(43-80)$ & $69.1(56-75)$ \\
\hline BMI & $26.8(19-35)$ & $26.4(19-32)$ & $28(24-35)$ \\
\hline CCI & $5.4(2-10)$ & $5.4(2-10)$ & $5.5(4-7)$ \\
\hline FEV1 (\%) & $86.2(44-126)$ & $87.7(44-113)$ & $82.7(57-126)$ \\
\hline $\begin{array}{l}\text { Surgical time (min- } \\
\text { utes) }\end{array}$ & $83.3(55-130)$ & $81.0(55-110)$ & $89.2(60-130)$ \\
\hline Drainage time (days) & $2.4(1-10)$ & $2.2(1-10)$ & $2.8(1-6)$ \\
\hline $\begin{array}{l}\text { Right upper lobec- } \\
\text { tomy }\end{array}$ & 2 & 0 & 2 \\
\hline $\begin{array}{l}\text { Right middle lobec- } \\
\text { tomy }\end{array}$ & 2 & 2 & 0 \\
\hline $\begin{array}{l}\text { Right lower lobec- } \\
\text { tomy }\end{array}$ & 9 & 7 & 2 \\
\hline Left upper lobectomy & 4 & 3 & 1 \\
\hline Left lower lobectomy & 3 & 3 & 0 \\
\hline Segmentectomy & 2 & 1 & 1 \\
\hline Wedge resection & 4 & 3 & 1 \\
\hline
\end{tabular}

CCI Carlson Comorbidity Index, BMI body mass index, FEVI Forced exspiratory volume in $1 \mathrm{~s}, S V I$ spontaneous ventilation combined with intubation, VATS video-assisted thoracic surgery information is included in this article If the patients agreed to this method, written informed consent was obtained. Patient data were retrieved retrospectively from our medical record system (Medsolution) and the patients' personal data were secured. The Ethical Committee of the Human Investigation Review Board in the University of Szeged approved this study (permission no.: 4703/2020.01.20).

\section{Patient selection}

The inclusion criteria are similar to the indications for the NITS, however, several exclusion criteria of the NITS (suspicion of difficult intubation, full anticoagulation, reflux disease, cardiac instability, mental problem) can be omitted [5]. In our current practice, patients with body mass index (BMI) of $<30$ are indicated for SVI.

Oncologically, the patient's selections for VATS SVI was performed as for normal VATS. According to a consensus meeting recommendations, patients without advanced stage lung cancer $(<7 \mathrm{~cm}, \mathrm{~N} 0$, and N1 patients) were scheduled [10]. For open SVI, we accepted simple cases, i.e. extended resections, and patients who underwent neoadjuvant treatment were excluded.

\section{Surgical procedure: VATS SVI}

Regarding the surgical steps, the same VATS uniportal method was performed as we published in our VATS NITS paper [11], but at the beginning of the surgery for a short time, the patients were relaxed and intubated with a double-lumen tube. At the incision site of the fifth intercostal space in the middle axillary line, $2 \%$ lidocaine $(5 \mathrm{mg} / \mathrm{kg})$ was administered. After entering into the thoracic cavity, vagus and intercostal nerve blockades were performed. The total amount of bupivacaine used was $0.5 \mathrm{ml} / \mathrm{kg}$ : $3-5 \mathrm{ml}$ of $0.5 \%$ bupivacaine was administered near the vagus nerve (right side in the upper mediastinum; left side in the aortopulmonary window) (Fig. 1.) and 4-5 ml near each intercostal nerve between 2 and 5, close to the spine (paravertebral blockade). Due to this local anaesthesia, if the relaxation stopped, the patient could spontaneously ventilate without
Fig. 1 Vagus blockade on right and left sides. $A A$ aortic arch, $N$ needle for injection, $P h N$ phrenic nerve, $S V C$ superior vena cava, $T$ trachea, $V N$ vagus nerve
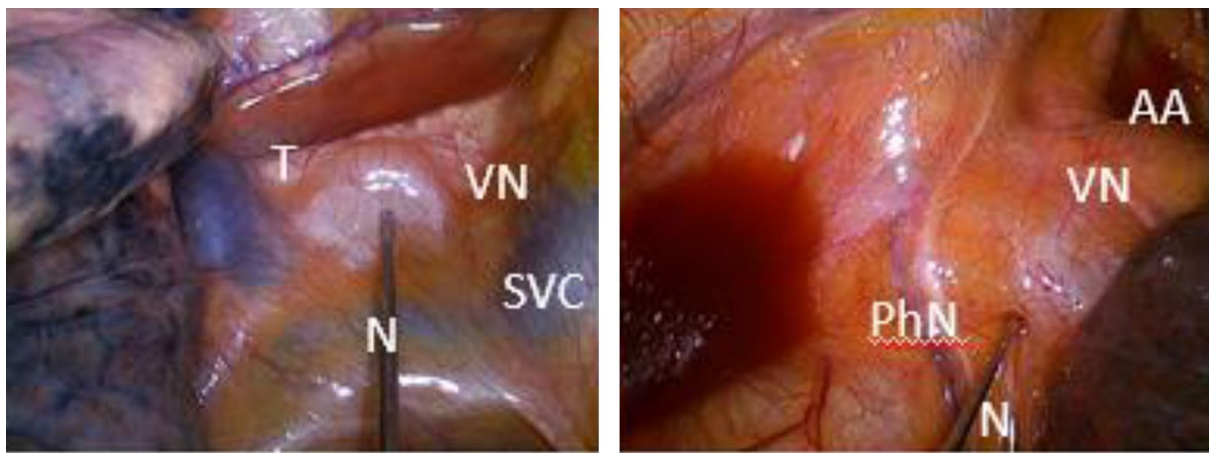
cough, and the mediastinal and diaphragmatic movements did not disturb during the resection. After the resection, the chest was drained and closed. The types of VATS SVI resections are listed in Table 1.

\section{Surgical procedure: open SVI}

In open cases, the patient was relaxed for a short time and intubated with a double-lumen tube. During the thoracotomy, an intercostal nerve blockade was performed using 4-5 $\mathrm{ml}$ of $0.5 \%$ bupivacaine between the 3.-6. ribs. After entering into the chest cavity, the same vagus nerve blockade was performed as in VATS SVI. After stopping the relaxation and resuming spontaneous breathing, all resections and mediastinal lymph node dissections could be performed. After the resection, the chest was drained and closed. The types of open SVI resections are presented in Table 1.

\section{Anaesthesiology}

The standard monitoring (ECG, $\mathrm{O}_{2}$ saturation, and depth of anaesthesia monitoring by bispectral index (BIS, Medtronic Vista) and invasive blood pressure measurements were performed. Preoperatively, midazolam and fentanyl were administered. Anaesthesia was induced and maintained with propofol, administered via the target-controlled infusion titrated to keep the BIS between 40 and 60, according to recommendations [9].

After the induction, the gold standard intubated technique for muscle relaxation and mechanical ventilation was performed. The difference in the period of muscle relaxation was determined using mivacurium instead of rocuronium, and the spontaneous breathing was allowed after eliminating the muscle relaxant. This ensured adequate time to perform vagal blockade to prevent cough reflex. After the return of spontaneous breathing, the patient did not cough due to regional blockades despite the double-lumen tube in the trachea. The oxygen saturation and $\mathrm{pCO}_{2}$ were kept within normal or close to the normal range with higher $\mathrm{FIO}_{2}$ (40-100\%) with 3-5 positive end-expiratory pressure in the dependent lung, which was easily measured through the double-lumen tube.

All of our patients have been observed in the post anaesthesia care unit for at least $2 \mathrm{~h}$ and until their visual analogue scale (VAS) went under 3. Oxygen was administered all of them via face mask usually with $2-41 /$ min to exceed $94 \%$ of oxygen saturation and $88 \%$ for patients with severe chronic obstructive pulmonary disease (COPD). Oxygen support was carried on with the same algorithm in the ward for 2-3 h. None of our patients required a higher level of oxygen support or advanced non-invasive respiratory support in the post anaesthesia care unit and later in the postoperative period.
Some patients, during the operation had hypotensive periods that might have been caused by the vagal blockade and its negative chronotropic effects. According to our protocol, we give $50-100 \mathrm{mcg}$ phenylephrine in divided dose if systolic blood pressure goes down below $100 \mathrm{mmHg}$ or decreases more than $25 \%$ or the mean arterial pressure is less than $60 \mathrm{mmHg}$.

\section{Inflammatory response}

On the preoperative-, and first- and second postoperative days (POD1; POD2) the inflammatory response to SVI was measured among six patients with leucocyte and lymphocyte counts, and level of C-reactive protein.

\section{Results}

\section{Surgery}

No perioperative mortality occurred. There were 2 conversions from SVI to relaxation method: in a patient, who underwent open SVI left sleeve 6 segmentectomy because of a $1 \mathrm{~cm}$ endobronchial lesion in the origin of the left 6 . segmental bronchus, due to the preparation and anastomosis suture, and in one other patient who underwent a conversion from SVI VATS to relaxation open lobectomy due to a bleeding. Therefore, the conversion rate from SVI to relaxation is $7.7 \%(2 / 26)$. Without changing from SVI to relaxation two additional conversions occurred. With keeping the SVI method, VATS approach was changed to open surgery due to oncological/technical reasons.

After terminating the relaxation, the patients breathe spontaneously, the diaphragm and the mediastinum movement were not disturbing, and surgeries were the same as in NITS cases. Perioperative data are presented in Table 1. The surgical and drainage times were 83.3 (55-130) minutes and 2.4 (1-10) days, respectively. The length of postoperative hospital stay was 4.1 (2-7) days. One patient went home with Heimlich-valve on the $7^{\text {th }}$ postoperative day. The mean number of the removed mediastinal lymph nodes is 9.8 (1-22). 16 primary lung cancers, 4 metastasis and 6 benign lesions were removed (Table 2.). In the 16 lung cancer cases, the mean number of the removed mediastinal lymph nodes is $10.1(1-22)$ : in a patient with removed brain metastasis from lung cancer, an SVI VATS wedge resection with hilar lymph node dissection and mediastinal sampling with 1 subcarinal mediastinal lymph node removal was performed (pT1aN1M1b). For the patient with 22 removed mediastinal lymph nodes, an open SVI right lower lobectomy was performed after neoadjuvant treatment (ypT1bN0) Data of the 20 patients who underwent lobectomies were presented in the Table 3. 
Table 2 Pathological results of the SVI resections

\begin{tabular}{|c|c|c|c|}
\hline & $\begin{array}{l}\text { All cases } \\
n=26\end{array}$ & $\begin{array}{l}\text { VATS SVI } \\
n=19\end{array}$ & $\begin{array}{l}\text { Open SVI } \\
n=7\end{array}$ \\
\hline Lung cancers & $n=16$ & $n=10$ & $n=6$ \\
\hline Adenocarcinoma & 7 & 6 & 1 \\
\hline Squamous cell carcinoma & 6 & 2 & 4 \\
\hline Large cell neuroendocrine carcinoma & 1 & 1 & 0 \\
\hline Carcinoid (typical) & 1 & 1 & 0 \\
\hline Carcinosarcoma & 1 & 0 & 1 \\
\hline Lung metastases & $n=4$ & $n=4$ & $n=0$ \\
\hline Colorectal carcinoma metastasis & 2 & 2 & 0 \\
\hline Melanoma metastasis & 1 & 1 & 0 \\
\hline Urinary bladder carcinoma metastasis & 1 & 1 & 0 \\
\hline Benign lesion & $n=6$ & $n=5$ & $n=1$ \\
\hline Tubecrulosis & 1 & 1 & 0 \\
\hline Actinomycosis & 1 & 1 & 0 \\
\hline Inflammation & 2 & 1 & 1 \\
\hline Hamartochondroma & 1 & 1 & 0 \\
\hline Sarcoidosis & 1 & 1 & 0 \\
\hline Stages of the lung cancers & $n=16$ & $n=10$ & $n=6$ \\
\hline IA & 7 & 4 & 3 \\
\hline IB & 2 & 2 & 0 \\
\hline IIA & 1 & 1 & 0 \\
\hline IIB & 2 & 2 & 0 \\
\hline IIIA & 3 & 0 & 3 \\
\hline IVA (with removed brain metastasis) & 1 & 1 & 0 \\
\hline
\end{tabular}

SVI spontaneous ventilation combined with intubation, VATS video-assisted thoracic surgery

\begin{tabular}{lll}
\hline & $\begin{array}{l}\text { VATS SVI lobectomies } \\
n=15\end{array}$ & $\begin{array}{l}\text { Open SVI lobectomies } \\
n=5\end{array}$ \\
\hline Male/female & $8 / 7$ & $4 / 1$ \\
Age & $62.4(46-79)$ & $69.6(56-75)$ \\
BMI & $25.6(22-32)$ & $28(24-35)$ \\
CCI & $4.7(2-8)$ & $5.2(4-7)$ \\
Surgical time (minutes) & $85.6(55-110)$ & $86(60-105)$ \\
Drainage time (days) & $2.2(1-10)$ & $2.4(1-4)$ \\
Postoperative length of hospital stay & $3.7(2-7)$ & $4.8(4-6)$ \\
$\quad$ (days) & & $74(57-104)$ \\
FEV1 (\%) & $88.7(78-113)$ & 2 \\
Right upper & 0 & 0 \\
Right middle & 2 & 2 \\
Right lower & 7 & 1 \\
Left upper & 3 & 0 \\
Left lower & 3 &
\end{tabular}

$B M I$ body mass index, $C C I$ Carlson Comorbidity Index, FEVI Forced exspiratory volume in $1 \mathrm{~s}, S V I$ spontaneous ventilation combined with intubation, VATS video-assisted thoracic surgery
Table 3 Results of the lobectomies in the SVI patients
None of our patients were observed in the ICU, and all of them were transported to the ward after a short observation in the post anaesthesia care unit. All the postoperative periods were uneventful, the patients could cough, and none of them had a fever or required bronchial cleaning. All the postoperative chest X-ray reports of all patients, before and after the chest tube removal were studied, and if any of them mentioned any size of atelectasis/dystelectasis, infiltration, 
pneumothorax or pleural fluid in the chest, the results were respected as an abnormal finding. Of the 26 patients, in 7 cases dystelectasis, in 0 case atelectasis, in 1 case infiltration, in 13 cases pneumothorax and in 13 cases pleural fluid were mentioned. None of the above-mentioned abnormalities required surgical interventions, such as drainage or puncture. There were 2 prolonged air leak cases (7.7\%) (6 and 10 days). No readmission or intervention was necessary for the follow-up period.

\section{Anaesthesiology}

About 5-10 min after the vagus nerve blockade, the blood pressure decreased; however, only 9 of 26 (34\%) patients were supported with phenylephrine. The remaining 15 patients had only temporary hypotension.

In the 26 cases, the mean mechanical one-lung ventilation time was $25.5(15-115)$ minutes, and thereafter, the mean spontaneous one-lung ventilation time was 73.3 (45-100) minutes, so the mechanical OLV time was reduced with $76.6 \%$ (50-87). In $92.3 \%$ of patients (24/26), only the initial relaxation drug for the intubation was required (Table 4.)

Hypercapnia was kept in a tolerable range, without any complication such as respiratory acidosis, right ventricular failure, or increased intracranial pressure. The mean maximal $\mathrm{pCO}_{2}$ was 52.3 (38-66) $\mathrm{Hgmm}$ and the mean lowest oxygen saturation was 93.8 (86-99)\%. (Table 4.)

The breathing frequency could be maintained for 10-25/ min with opioid administration. Postoperatively, all patients were extubated without using an acetylcholinesterase inhibitor and after an hour of observation in the recovery room, they were transported to the general ward.

None of them required re-intubation.

\section{Inflammatory response}

During the POD1 leucocyte count increased with $11 \%$, however, on the POD2 it dropped (88\%) below the preoperative level. Very limited change was seen in the lymphocyte count: on the POD1 it was almost the same as preoperatively (98\% vs 100\%), however, on the POD2 it was higher than the preoperative level (119\%). Compared to baseline measures, on POD1 CRP level elevated to $231 \%$, however, it did not significantly change on POD2 (235\%). (Table 5.)

\section{Discussion}

The SVI procedure was developed as the combination of the NITS/spontaneous ventilation thoracic surgery and intubated thoracic procedures. The key point of the SVI is the vagus nerve blockade with bupivacaine $[9,11]$. With this local anaesthesia, the function of the vagus is blocked, and the cough reflex is suspended during the lung resection [8]. If the patient came out from the initial relaxation administered due to intubation, they can breathe spontaneously without any cough, and from this point, the surgery is the same as in NITS cases. It was not necessary to repeat the bupivacaine for the vagus blockade during the 73.3 min mean spontaneous one-lung ventilation time.
Table 4 Anaesthesiologic parameters during SVI surgeries

Table 5 Inflammatory parameters during SVI surgeries

\begin{tabular}{|c|c|c|c|c|c|c|c|c|c|}
\hline \multicolumn{2}{|c|}{ Pulse/minute } & \multirow[t]{2}{*}{$\begin{array}{l}\text { Mech OLV } \\
\text { (minute) }\end{array}$} & \multirow{2}{*}{$\begin{array}{l}\text { Spontan } \\
\text { OLV (min- } \\
\text { ute) }\end{array}$} & \multicolumn{2}{|c|}{$\begin{array}{l}\text { Oxygen satura- } \\
\text { tion } \%\end{array}$} & \multicolumn{2}{|l|}{$\begin{array}{l}\mathrm{pCO} 2 \\
\text { Hgmm }\end{array}$} & \multicolumn{2}{|c|}{ Breath/minute } \\
\hline Min & Max & & & Min & Max & Min & Max & Min & Max \\
\hline 66.4 & 84.9 & 25.5 & 73.3 & 93.8 & 99.3 & 39.1 & 52.3 & 12.1 & 18.3 \\
\hline $48-90$ & $65-120$ & $15-115$ & $45-100$ & $86-99$ & $98-100$ & $28-50$ & $38-66$ & $10-15$ & $14-25$ \\
\hline
\end{tabular}

SVI spontaneous ventilation combined with intubation, Max maximal, Mech Mechanical, min. minimal, $O L V$ one-lung ventilation, $p C O 2$ carbon dioxide pressure, spontan. spontaneous

\begin{tabular}{llll}
\hline & Preoperative $n=6$ & $\begin{array}{l}\text { Postoperative } \\
24 \mathrm{~h} \\
n=6\end{array}$ & $\begin{array}{l}\text { Postoperative } 48 \mathrm{~h} \\
n=6\end{array}$ \\
\hline Leucocytes $\left(10^{9} / \mathrm{L}\right)$ & $10.9(100 \%)$ & $12.1(111 \%)$ & $9.6(88 \%)$ \\
& $(7.1-13.8)$ & $(10.1-15.9)$ & $(8.3-10.4)$ \\
Lymphocytes $\left(10^{9} / \mathrm{L}\right)$ & $1.31(100 \%)$ & $1.29(98 \%)$ & $1.56(119 \%)$ \\
& $(0.38-2.25)$ & $(0.87-1.7)$ & $(1.07-1.82)$ \\
CRP $(\mathrm{mg} / \mathrm{L})$ & $29.6(100 \%)$ & $68.6(231 \%)$ & $69.7(235 \%)$ \\
& $(18.2-44.0)$ & $(61.1-72.9)$ & $(49.7-82.0)$ \\
\hline
\end{tabular}

CRP C-reactive protein, $L$ liter, VATS video-assisted thoracic surgery, SVI spontaneous ventilation combined with intubation 
Local anesthesia in thoracic surgery is not a new method. Ossipov [12] presented 3265 thoracic interventions under local anesthesia. He performed an extended infiltration in the following sequence. First, he performed vagosympathetic blockade on the neck and then wide local infiltration in the skin incision, followed by infiltration under the scapula, blockade of the Th2-Th9 intercostal and sympathetic nerves, and finally, the same vagus blockade that is used in the NITS procedures [9] and in the SVI.

Any risk of SVI can be temporary if it occurs. In the period of spontaneous one-lung ventilation, a double-lumen tube is located in its properly placed, giving chance to all interventions and medications as the situation requires.

If any surgical difficulty or complication occurred, the patient could be relaxed and the surgery would continue under a mechanical one-lung ventilation, as a "normal thoracic surgery', and the uncomfortable conversion procedure (0-9\% of the cases) [13] from NITS to intubation is prevented with the SVI. Moreover, as demonstrated, the relaxation was not absolutely necessary for thoracotomy: six open thoracotomies were performed with the SVI method.

Regarding postoperative results, mean operative- and drainage time was shorter after our SVI VATS lobectomy cases compared to AlGhambi's study on non-intubated and intubated/relaxed VATS lobectomies: 85.6, 130.9 and $146.0 \mathrm{~min}$ and 2.2, 5.6 and 5.4 days, respectively [14]. In our study, the mean postoperative length of hospital stay after VATS SVI and open SVI lobectomies were 3.7 and 4.8 days, which is shorter than the same data in the abovementioned study after non-intubated and intubated/relaxed VATS lobectomies: 6.9 and 7.6 days [14].

In VATS NITS/SV patients, a disturbing mediastinal and diaphragmatic movements were the most common complication requiring intubation, occurring in $7 \%$ and $4 \%$ of a meta-analysis article in Shi et al. [15]. In our short series of SVI, this kind of disturbing movement rate was $1 / 26(2.8 \%)$ requiring conversion from SVI to the relaxation and permanent mechanical one-lung ventilation (4 weeks later a smooth SVI VATS lobectomy was performed on the other side for this patient). The other conversion to relaxation performed due to a bleeding, from a segmental artery demanding thoracotomy. However, other $92.3 \%$ did not require additional relaxation after the induction. We had no conversion from SVI to relaxation due to oncological reason (only the previously mentioned technical reason and bleeding). The 2 conversion from VATS SVI to open SVI happened due to oncological/technical reason without any complication; however, the patients required 200 and 400 micg of phenylephrine as the circulation support. The postoperative periods were smooth.

If any anaesthesiologic difficulties occur, additional oxygen support, air-way cleaning, or relaxation can be performed in seconds due to the double-lumen tube located in the trachea and main bronchus, and all problems can be managed on a 'traditional way'. We have seen the same hypercapnia and hypoxia during the SVI as in patients with NITS [15]; however, it was easy to compensate with higher $\mathrm{FIO}_{2}$ through the double-lumen tube.

At 5-10 min after the vagus blockade, the blood pressure was reduced, but only in $34 \%$ of the patients required circulation support. In the remaining $66 \% \mathrm{~s}$, the self-regulation of the circulation normalized the blood pressure.

With the SVI, we could reduce the period of mechanical OLV by $76.6 \%$. Misthos et al. reported that mechanical OLV for longer than $1 \mathrm{~h}$ could be a reason for the severe oxidative stress [6]. The OLV lung plays a major role in oxidative stress [3] and in SVI cases the reduced mechanical OLV time may produce less changes in lung physiology. In our cases, the tendency of inflammatory response to SVI was similar to the data of Mineo [3]. Among our patients, mean leucocyte count elevated on the POD1 to $111 \%$, which was $138 \%$ among non-intubated and intubated VATS cases in Mineo's reports. On the POD1, mean lymphocyte count decreased to $98 \%$ among our patients, which data showed the same pattern with $92 \%$ and $83 \%$ in non-intubated and intubated cases of Mineo [3]. It seems, that the inflammatory response to SVI may be very close to the response after non-intubated procedures. Although in the study of Leaver, changes in lymphocyte count were not detected on POD1 and the highest deviation from baseline was detected on POD2 [16], our study with SVI cases showed that the leucocyte- and lymphocyte counts on POD2 are closer to baseline after the highest deviation seen on POD1.

In our study, the CRP levels doubled on POD1 and did not show significant elevation on POD2. But in the study of Dongel, patients undergoing thoracotomy and intubation with relaxation, CRP levels elevated until reaching $425 \%$ on POD1 and 353\% on POD3, compared to baseline level [17].

With the SVI procedure, the indications of the spontaneous ventilation thoracic procedures can be more widespread, and because of the safe airway with the double-lumen tube, several previous exclusion criteria of the NITS can be accepted as an indication criteria for SVI: abnormal airway anatomy and anticipated difficult intubation, reflux disease, risk of blood or other fluid in the airway, mental problem [5, 9]. More patients can be selected for spontaneous ventilation surgery.

\section{Conclusion}

The SVI technique is a safe method for VATS and open thoracic surgeries for patients with BMI of $<30$. There are less exclusion criteria for SVI than for NITS. The SVI could reduce the period of mechanical OLV with $77 \%$. No complications were observed in patients during the SVI surgery 
because of the safe airway with a double-lumen tube that provides chance for all anaesthesiologic procedures as it is in normally relaxed patients, and the conversion can be very simple. After the vagus blockade, no cough occurred at all despite the double-lumen tube location in the trachea and main bronchus. Regarding leucocyte- and lymphocyte counts, the inflammatory response in SVI cases is very similar to the effect of non-intubated procedures.

Acknowledgements We would like to thank Editage (www.edita ge.com) for English language editing.

\section{Compliance with ethical standards}

Conflict of interest The authors declare no conflicts of interest: FJ has no conflict of interest; SzZs has no conflict of interest.

\section{References}

1. Gonzalez-Rivas D, Fernandez R, de la Torre M, Rodriguez JL, Fontan L, Molina F. Single-port thoracoscopic lobectomy in a nonintubated patient: the least invasive procedure for major lung resection? Interact Cardiovasc Thorac Surg. 2014;19:552-5.

2. Liu J, Cui F, Pompeo E, Gonzalez-Rivas D, Chen H, Yin W, et al. The impact of non-intubated versus intubated anaesthesia on early outcomes of video-assisted thoracoscopic anatomical resection in non-small-cell lung cancer: a propensity score matching analysis. Eur J Cardiothorac Surg. 2016;50:920-5.

3. Mineo TC, Ambrogi V. Immune effects after uniportal nonintubated video-thoracoscopic operations. Video-assist Thorac Surg. 2018;3:4-10.

4. Lohser J, Slinger P. Lung injury after one-lung ventilation: a review of the pathophysiologic mechanisms affecting the ventilated and the collapsed lung. Anesth Analg. 2015;121:302-18.

5. Gonzalez-Rivas D, Bonome C, Fieira E, Aymerich H, Fernandez $\mathrm{R}$, Delgado M, et al. Non-intubated video-assisted thoracoscopic lung resections: the future of thoracic surgery? Eur J Cardiothorac Surg. 2016;49:721-31.

6. Misthos P, Katsaragakis S, Theodorou D, Milingos N, Skottis I. The degree of oxidative stress is associated with major adverse effects after lung resection: a prospective study. Eur J Cardiothorac Surg. 2006;29:591-5.

7. Magnusson L. Role of spontaneous and assisted ventilation during general anaesthesia. Best Pract Res Clin Anaesthesiol. 2010;24:243-52.

8. Polverino M, PolverinoF FM, Andò F, Alfieri A, De Blasio F. Anatomy and neuro-pathophysiology of the cough reflex arc. Multidiscip Respir Med. 2012;7:5.

9. Hung MH, Hsu HH, Chan KC, Chen KC, Yie JC, Cheng YJ, et al. Non-intubated thoracoscopic surgery using internal intercostal nerve block, vagal block and targeted sedation. Eur J Cardiothorac Surg. 2014;46:620-5.

10. Yan TD, Cao C, D’Amico TA, Demmy TL, He J, Hansen H, et al. Video-assisted thoracoscopic surgery lobectomy at 20 years: a consensus statement. Eur J Cardiothorac Surg. 2014;45:633-9.

11. Furák J, Szabó ZS, Tánczos T, Paszt A, Rieth A, Németh T, et al. Conversion method to manage surgical difficulties in non-intubated uniportal video-assisted thoracic surgery for major lung resection: simple thoracotomy without intubation. J Thorac Dis. 2020;2:2061-9.

12. Ossipov BK. Local anesthesia in thoracic surgery: 20 years experience with 3265 cases. Anesth Analg. 1960;39:327-32.

13. Mineo TC, Tacconi F. From, "awake" to "monitored anesthesia care" thoracic surgery: a 15 year evolution. Thorac Cancer. 2014;5:1-13.

14. AlGhamdi ZM, Lynhiavu L, Moon YK, Moon MH, Ahn S, Kim $\mathrm{Y}$, et al. Comparison of non-intubated versus intubated videoassisted thoracoscopic lobectomy for lung cancer. J Thorac Dis. 2018;10:4236-43.

15. Shi Y, Yu H, Huang L, Wang S, Chi D, Chen CH, et al. Postoperative pulmonary complications and hospital stay after lung resection surgery. A meta-analysis comparing nonintubated and intubated anesthesia. Medicine (Baltimore). 2018;97:e10596.

16. Leaver HA, Craig SR, Yap PL, Walker WS. Lymphocyte responses following open and minimally invasive thoracic surgery. Eur J Clin Invest. 2000;30:230-8.

17. Dongel I, Gokmen AA, Gonen I, Kaya S. Pentraxin-3 and inflammatory biomarkers related to posterolateral thoracotomy in Thoracic Surgery. Pak J Med Sci. 2019;35:464-9.

Publisher's Note Springer Nature remains neutral with regard to jurisdictional claims in published maps and institutional affiliations. 